\title{
Review of: "Meta-analysis of the efficacy of preoperative skin preparation with alcoholic chlorhexidine compared to povidone iodine in orthopedic surgery"
}

\author{
Hideto Yasuda
}

Potential competing interests: The author(s) declared that no potential competing interests exist.

One of the important aspects of systematic reviews and meta-analyses is whether high quality systematic review and meta-analysis methods are used; whether external and internal clinical validity is ensured is also important. The present study has several problems related to the above two points.

1. It is important for systematic reviews to show reproducibility. In this paper, the key words are presented, but the search formula to ensure reproducibility is not presented, and the validity of the search cannot be evaluated.

2. An important aspect of systematic reviews and meta-analyses is not only to provide point estimates of effects and their confidence intervals, but also to assess the certainty of the evidence for those effects. In recent years, it is common to evaluate the results by the Grading of Recommendations, Assessment, Development and Evaluation (GRADE) system, ${ }^{[1]}$ and if the certainty of evidence is not shown by GRADE, the certainty of the effect cannot be evaluated and the external validity is reduced.

3. The effects of chlorhexidine vary with concentration. In the world, $2 \%$ chlorhexidine alcohol is generally used, but in some countries, such as Japan, it can only be used up to a $1 \%$ concentration. Chlorhexidine is used clinically in concentrations ranging from $0.5 \%$ to $2 \%$. However, a network meta-analysis of the effects on the prevention of catheter-related blood stream infection (CRBSI) reported by our research group $^{[2]}$ showed that $0.5 \%$ chlorhexidine alcohol was less effective than a highly concentration of chlorhexidine alcohol against CRBSI. This study should be meta-analyzed with a design that allows for the examination of differences in chlorhexidine concentrations, as was the approach taken in our study.

4. One way to check whether or not the sample size required to ensure the validity of the results has been reached is to evaluate it by imprecision with GRADE or to perform a trial sequential analysis. Since neither of these methods was confirmed in this study, it is not possible to evaluate the reason for the lack of significant results.

5. The primary outcome is culture of indigenous skin bacteria, but it is questionable whether this is a clinically important outcome. The primary outcome for systematic review and meta-analysis should be set to a clinically important outcome.

References

1. Guyatt G, Oxman AD, AkI EA, et al. GRADE guidelines:383-394. GRADE guidelines: 1. Introduction- 
GRADE evidence profiles and summary of findings tables. J Clin Epidemiol 2011 Apr;64(4):383-394.

2. Masuyama T, Yasuda H, Sanui M, et al. Effect of skin antiseptic solutions on the incidence of catheter-related bloodstream infection: a systematic review and network meta-analysis. J Hosp Infect 2021 Apr;110:156-164. 